\title{
„Man höre diesem Nietzsche eindringlicher zu, als dem, der anders spricht."
}

Heinrich Manns Auseinandersetzung mit nationalsozialistischen Nietzsche-Mythen

"Écoutez ce Nietzsche plus attentivement que ceux qui tiennent un autre langage. " La confrontation de Heinrich Mann avec les mythes nationauxsocialistes autour de Nietzsche

"One should listen more attentively to this Nietzsche than to those who speak differently." Heinrich Mann's Confrontation with National Socialist Nietzsche Myths

\section{Cordula Greinert}

\section{(2) OpenEdition}

\section{Journals}

Édition électronique

URL : http://journals.openedition.org/ceg/4345

DOI : $10.4000 /$ ceg.4345

ISSN : 2605-8359

Éditeur

Presses Universitaires de Provence

Édition imprimée

Date de publication : 2 mai 2019

Pagination : 63-78

ISBN : 979-10-320-0214-8

ISSN : 0751-4239

\section{Référence électronique}

Cordula Greinert, "m,Man höre diesem Nietzsche eindringlicher zu, als dem, der anders spricht."', Cahiers d'Études Germaniques [Online], 76 | 2019, Online erschienen am: 02 November 2020, abgerufen am 24 Januar 2021. URL: http://journals.openedition.org/ceg/4345 ; DOI: https://doi.org/10.4000/ceg.4345 


\section{„Man höre diesem Nietzsche eindringlicher zu, als dem, der anders spricht. ${ }^{6}$}

Heinrich Manns Auseinandersetzung mit nationalsozialistischen Nietzsche-Mythen

\section{Cordula GREINERT}

Johannes Gutenberg-Universität Mainz

Die publizistische Auseinandersetzung mit dem nationalsozialistischen Deutschland und den in ihm verbreiteten Mythen war für Heinrich Mann in seiner französischen Exilzeit täglich Brot. Seine ca. 400 Texte aus den Jahren 1933 bis 1940 füllen drei Bände der neunbändigen Kritischen Gesamtausgabe seiner Essays und Publizistik. ${ }^{1}$ Das Spektrum reichte von regelmäßigen Beiträgen in Exilzeitschriften und französischen Tageszeitungen bis $\mathrm{zu}$ gelegentlichen Aufrufen, Manifesten und Interviews. Nur in einem Fall verfasste er in dieser Zeit einen umfänglichen Personenessay ${ }^{2}$ - diese Ausnahme betrifft einen der umstrittensten Philosophen seiner Zeit: Friedrich Nietzsche.

Jener Essay entstand 1938 auf Anfrage des österreichisch-jüdischen Werbefachmanns und Publizisten Alfred O. Mendel, der 1936 in die USA emigriert war, wo er sich als Literaturagent zu etablieren versuchte. In einem Brief aus New York vom 31. August 1937 teilte er Heinrich Mann mit, dass er im Begriff sei,

eine Buchreihe auszuarbeiten, die den Zweck hat, gewisse vielzitierte und weniggelesene Werke der Weltliteratur (im weitesten Sinn) in ihren Hauptzuegen dem amerikanischen Publikum vorzufuehren. Doch will ich nicht etwa mit dem Neudruck alter Werke, sondern mit ihrer sozusagen zeitgemaessen Wiederbelebung beginnen, - mit dem, was nach heutigem Masstab unsterblich an ihnen ist. ${ }^{3}$

Die Bücher sollten in dem in den USA populären Digest-Format erscheinen. Die Herausgabe des Nietzsche-Bandes trug Mendel Heinrich Mann an und bat ihn,

1. Vgl. Heinrich Mann, Essays und Publizistik. Kritische Gesamtausgabe, hrsg. von Wolfgang Klein, Anne Flierl und Volker Riedel, Bd. 6: Februar 1933 bis 1935, hrsg. von Wolfgang Klein mit Vorarbeiten von Werner Herden, Bielefeld, Aisthesis, 2009; Bd. 7: 1936 bis 1937, hrsg. von Wolfgang Klein (in Vorbereitung); Bd. 8: 1938 bis September 1940, hrsg. von Cordula Greinert (in Vorbereitung).

2. Seine früheren großen Personenessays widmete er französischen Schriftsteller*innen; vgl. Heinrich Mann, „Eine Freundschaft. Gustave Flaubert und George Sand“ (1905/06) sowie „Zola“ (1915), in ders., Essays und Publizistik, Bd. 2: Oktober 1904 bis Oktober 1918, hrsg. von Manfred Hahn unter Mitarbeit von Anne Flierl und Wolfgang Klein, Bielefeld, Aisthesis, 2012, S. 33-64, 148-209.

3. Alfred O. Mendel an Heinrich Mann, New York, 31. August 1937 (University of Southern California, Los Angeles, Special Collections, Feuchtwanger Memorial Library [FML], Heinrich Mann Collection, Box 6, File 14). 
dafür sowohl Auszüge aus Nietzsches Werken zusammenzustellen als auch eine Einleitung zu schreiben. Zur Autonomie des Autors hielt er fest: „Mir ist es um das heutige Bild eines frueheren Denkers zu tun: wie Sie es sehen! Man soll nicht sagen: ,Das ist Nietzsche“, sondern ,So sieht Heinrich Mann Nietzsche!،“44 1939 kam das Buch auf Englisch in der „Living Thoughts“ betitelten Reihe heraus. ${ }^{5}$ In deutscher Sprache erschien Heinrich Manns Einleitung zudem in der von Thomas Mann und Konrad Falke in Zürich herausgegebenen Zeitschrift Mass und Wert, ${ }^{6}$ die jedoch einige längere Auslassungen sowie mehrere - trotz Fahnenkorrektur - im Text verbliebene sinnentstellende Druckfehler aufweist. Erst 53 Jahre später wurde der Essay vollständig nach der Handschrift ediert. ${ }^{7}$

Für den vorliegenden Artikel soll der Text daraufhin untersucht werden, wie Heinrich Mann sich zu einigen Theoremen Nietzsches positionierte, die entweder schon selbst mythisch aufgeladen oder aber mittlerweile zu Mythen transformiert und nationalsozialistisch usurpiert worden waren. Zu berücksichtigen ist hierbei insbesondere seine Rolle als intellektueller Repräsentant der 1938 akut im Scheitern begriffenen antifaschistischen Einigungsbestrebungen im Exil. Auch sein Kommentar, sein Aufsatz sei „,hauptsächlich für Amerika geschrieben“" vor dem Hintergrund der ,,adressatenbezogenen Schreibweise“" ${ }^{\text {"9 }}$ seiner Essayistik zu berücksichtigen sein.

\section{Begriffe und Kontexte}

Das hier zugrundeliegende Verständnis des Mythos ist das eines ideologischpolitischen Kampfbegriffs des 20. Jahrhunderts, der „nicht nur Irrationalitäten versammelt, sondern den Begriff selbst unbestimmt läßt und Inhalte der chauvinistisch gewendeten romantischen Nationalmythologien mit einem politischen Voluntarismus

4. Ibid.

5. The Living Thoughts of Nietzsche, presented by Heinrich Mann, New York/ Toronto, Longmans, Green, 1939. Für Großbritannien wurde eine separate Ausgabe gedruckt, zudem erschienen Übersetzungen in Frankreich (Les Pages immortelles de Nietzsche), Norwegen (Nietzsche), Argentinien (El Pensamiento Vivo de Nietzsche), Brasilien (O Pensamento Vivo de Nietzsche), Bulgarien (Bezsmărtnitě Misli na Nitče), Dänemark (Nietzsches Udødelige Tanker) und in den Niederlanden (De Levende Gedachten van Nietzsche) sowie Auszüge auf Koreanisch („Niich'e“). Von den insgesamt 21 Bänden der US-Reihe wurden viele von deutschen und österreichischen Exilanten oder französischen Schriftstellern herausgegeben, darunter diejenigen zu Schopenhauer (Thomas Mann), Montaigne (André Gide), Tolstoj (Stefan Zweig), Rousseau (Romain Rolland), Spinoza (Arnold Zweig), Voltaire (André Maurois), Marx (Lev Trotzkij und Otto Rühle), Pascal (François Mauriac), Konfuzius (Alfred Döblin), Kant (Julien Benda) und Freud (Robert Waelder).

6. Vgl. Heinrich Mann, „Nietzsche“, Mass und Wert. Zweimonatsschrift für freie deutsche Kultur, Jg. 2, Heft 3, Januar/Februar 1939, S. 277-304.

7. Vgl. Heinrich Mann, „Nietzsche“, in Nietzsches unsterbliche Gedanken. Eingeleitet von Heinrich Mann. Ausgewählt von Golo Mann, hrsg. und mit einem Nachwort von Wolfgang Klein, Berlin, Aufbau, 1992, S. (7)-43.

8. Heinrich Mann an Klaus Pinkus, 3. Januar 1939, in Heinrich Mann, Briefe an Karl Lemke und Klaus Pinkus, hrsg. von der Deutschen Akademie der Künste zu Berlin, Hamburg, Claassen, [1964], S. 139f., hier: 140.

9. Peter Stein, Heinrich Mann, Stuttgart/ Weimar, Metzler, 2012, S. 118. 
verbindet" ${ }^{10}$. Das suggestive Potenzial des Mythos wird dabei für politische Zwecke instrumentalisiert. Auf diese Weise werden unter anderem Herrschaftsansprüche legitimiert, Autorität begründet und Identität gestiftet - in der Regel um den Preis einer historisch oder ethnisch begründeten Abgrenzung von „Anderen“. Die damit einhergehende Reduktion gesellschaftlicher Komplexität zeitigt neben vorgeblich eindeutigen politischen Handlungsalternativen insbesondere eine Abwehr diskursiver Entscheidungsverfahren. Der politische Diskurs wird somit aus einem deliberativen Rahmen in einen mythisch-schicksalhaften verschoben. Da der moderne Mythos als eindeutig und unmissverständlich konstruiert wird, verliert er seine ursprüngliche Ambivalenz und sein damit verbundenes kritisches Potenzial. ${ }^{11}$

Nietzsches Werke und seine Person waren für ein solches Vorgehen in mehrerer Hinsicht prädestiniert: Zum einen boten die „Fülle suggestiver Themen, Ideen und Kategorien“ sowie „seine funkelnde Sprache und Rhetorik“ viele Anknüpfungspunkte für radikale Strömungen. Zum anderen war schon früh, nicht zuletzt aufgrund seiner Krankheit, ein Mythos um seine Person - ein NietzscheKult - entstanden. Doch während vor 1914 vornehmlich avantgardistische Gruppen seine Philosophie rezipierten, wurde sein Denken - insbesondere seine Begriffe von „Härte“ und „Heldentum“ - im Ersten Weltkrieg germanisiert und nationalisiert. Diese Entwicklung fiel zusammen mit einer „Modernisierung“ großer Teile der radikalen Rechten auf der Suche nach neuen Referenzen. ${ }^{12}$ Auch die weitere Rezeption von Nietzsches philosophischen Theoremen vollzog sich in erster Linie schlagwortinduziert und nicht auf Werkebene. Insbesondere das „Dionysische“, der „Tod Gottes“, der „Übermensch“, die „Umwertung aller Werte“, der „Wille zur Macht“ und die „ewige Wiederkehr des Gleichen“ fanden so ihren Weg in das ideologisch-politische Vokabular. ${ }^{13}$ Anders als im italienischen Faschismus, der Nietzsches Denken von Beginn an als eine seiner Grundlagen adaptierte, verlief die Rezeption im deutschen Nationalsozialismus weder überwiegend affirmativ noch sonderlich kenntnisreich. ${ }^{14}$ Dies verhinderte allerdings nicht, dass Nietzsche in das „Pantheon der germanischen Geistesgrößen“" ${ }^{15}$ eingereiht - und somit mythologisiert - wurde. Gleichzeitig wurden seine Ideen aus ihrem historischen Kontext des 19. in das 20. Jahrhundert transferiert und aktualisiert. ${ }^{16}$

10. Ernst Müller, „Mythos/ mythisch/ Mythologie“, in Karlheinz Barck, Martin Fontius, Dieter Schlenstedt, Burkhart Steinwachs, Friedrich Wolfzettel (Hrsg.), Ästhetische Grundbegriffe. Historisches Wörterbuch in sieben Bänden, Bd. 4: Medien-Populär, Studienausgabe, Stuttgart/ Weimar, Metzler, 2010 [2002], S. 309-346, hier: 336.

11. Vgl. Sabine Behrenbeck, Der Kult um die toten Helden. Nationalsozialistische Mythen, Riten und Symbole 1923 bis 1945, Vierow bei Greifswald, SH-Verlag, 1996, S. 44-46.

12. Vgl. Steven Aschheim, „Nietzsche“, in Etienne François, Hagen Schulze (Hrsg.), Deutsche Erinnerungsorte I, München, C. H. Beck, 2001, S. 502-519, hier: 502, 508, 511.

13. Vgl. Jochen Schmidt, Der Mythos ,,Wille zur Macht“. Nietzsches Gesamtwerk und der NietzscheKult. Eine historische Kritik, Berlin/ Boston, de Gruyter, 2016, S. 2-4.

14. Vgl. Hans-Martin Gerlach, „Politik (Faschismus, Nationalsozialismus, Sozialdemokratie, Marxismus)“, in Henning Ottmann (Hrsg.), Nietzsche-Handbuch. Leben - Werk - Wirkung, Stuttgart, Metzler, 2000/ 2011, S. 499-509, hier: 501.

15. Aschheim, „Nietzsche“, S. 515.

16. Vgl. Ibid. Zur Verfahrensweise der nationalsozialistischen Aktualisierung von Mythen vgl. Behrenbeck, Der Kult um die toten Helden, S. 47f. 
Eine maßgebliche Rolle hierfür spielte der Philosoph Alfred Baeumler, der - als neu berufener Professor für Politische Pädagogik an der Berliner Friedrich-WilhelmsUniversität - in seiner Antrittsvorlesung am 10. Mai 1933 die anschließende, von der Deutschen Studentenschaft organisierte, Bücherverbrennung auf dem Opernplatz ausdrücklich begrüßte. Als Herausgeber von Nietzsches Werken hatte er bereits 1930 Thesen wie jene aufgestellt, dass Nietzsche „eine Wiedergeburt der hellenischen Welt aus den tiefsten Instinkten des germanischen Wesens“" angedacht, „die ,Ausscheidung des Romanischen“ [...] als eine notwendige Folge der Heimkehr des deutschen Geistes zu sich selbst und seiner dionysischen Urheimat aufgefaßt" und „das Bild des heroischen Menschen, des Menschen eines Triebes, des Triebes zu kämpfen, zu siegen und im Siege sich zu opfern“"17, gezeichnet habe. Ein Jahr nach Antritt seiner Professur und nach Regierungsantritt der NSDAP parallelisierte Baeumler dann direkt Hitler und Nietzsche als einsame Schöpfer und Kämpfer - der eine auf der politischen, der andere auf der religiös-geistigen, ,gibhellinischen“ Linie einer „nordischen Bewegung“. Nietzsche für seinen Teil habe „,[a]n die Stelle der bürgerlichen Moralphilosophie [...] die Philosophie des Willens zur Macht, d. h. die Philosophie der Politik“ gesetzt. Sinn und Ziel einer solchen „,neuen Politik“ bestehe darin, den „Grundgegensatz“ zwischen dem „demokratische[n] Gleichheitsideal“ und „einem natürlichen Lebenszusammenhang" zugunsten von letzterem zu entscheiden: „Es liegt eine unerhörte Kühnheit in dem Unterfangen, den Staat auf die Rasse zu gründen. Eine neue Ordnung der Dinge muß sich daraus ergeben. Es ist jene Ordnung, die Nietzsche gegenüber der bestehenden wieder hat herstellen wollen.“ Er beendete seinen Text mit der Huldigung: „Heil Hitler! - so grüßen wir mit diesem Rufe zugleich Friedrich Nietzsche." ${ }^{18}$ Die mit dieser mythischen Überhöhung einhergehende Umdeutung von Nietzsches radikalem Individualismus als ,verwurzelt“ in einem - völkisch und rassistisch konkretisierten - Kollektiven, die Negierung historischer Unterschiede, die Konstruktion eines einheitlichen, systematischen Denkens bei Nietzsche sowie dessen Präsentation als Philosoph und Politiker ${ }^{19}$ ermöglichten es Baeumler, in dessen Schriften „den Weg zu einer neuen Staatslehre“" auszumachen. ${ }^{20}$

Diese Einschätzung von Nietzsche als Vorläufer des Faschismus wurde in weiten Kreisen der politischen Linken geteilt. Ausgehend von einer ideologiekritischen, historisch-materialistischen Analyse lehnten sie Nietzsche als „Philosoph des Kapitalismus“ (Franz Mehring), „Begründer des imperialistischen Irrationalismus“ (Georg Lukács) oder „kritischen Apologeten“ des Kapitalismus (Hans Günther) ab. Im Gegensatz dazu sahen radikaldemokratische Strömungen in Nietzsches

17. Alfred Baeumler, „Nietzsche“ (1930), in ders., Studien zur deutschen Geistesgeschichte, Berlin, Junker und Dünnhaupt, 1937, S. 244-280, hier: 250, 258, 259; Kursivierung im Original gesperrt.

18. Ders., „Nietzsche und der Nationalsozialismus“ (1934), in ders., Studien zur deutschen Geistesgeschichte, S. 281-294, hier: 283, 292, 294; Kursivierungen im Original gesperrt.

19. Vgl. ders., Nietzsche der Philosoph und Politiker, Leipzig, Reclam, 1931.

20. Giorgio Penzo, Der Mythos vom Übermenschen. Nietzsche und der Nationalsozialismus, übers. von Barbara Häußler, Frankfurt a. M. et al., Peter Lang, 1992, S. 160f. Vgl. zudem Philipp Teichfischer, Die Masken des Philosophen. Alfred Baeumler in der Weimarer Republik - eine intellektuelle Biographie, Marburg, Tectum, 2009. 
„revolutionärem Individualismus“ einen Ausgangspunkt für die Suche nach gemeinsamen Anknüpfungspunkten zur Überwindung der bestehenden Ordnung. Zwischen diesen beiden Polen bewegten sich verschiedene Ansätze, die beispielsweise darauf abstellten, dass der Sozialismus die Massen nicht verelenden, sondern - auch geistig - veredeln wolle (Kurt Eisner), die Nietzsches kritisches Potenzial gegen Spießer- und Kleinbürgertum betonten (Ernst Bloch) oder die in Nietzsche einen Dialektiker der Aufklärung sahen (Max Horkheimer und Theodor W. Adorno). ${ }^{21}$

Auch in Heinrich Manns Nachlassbibliothek finden sich differenzierende Stimmen zu Nietzsche. Aus Unterstreichungen, Marginalien und Notizen geht hervor, dass er neben einem Widmungsexemplar von Marius-Paulin Nicolas' De Nietzsche à Hitler ${ }^{22}$ Karl Jaspers' Band Nietzsche. Einführung in das Verständnis seines Philosophierens ${ }^{23}$ zu Rate zog, der Teil einer Büchersendung Golo Manns zur Vorbereitung seines Essays gewesen war. Nicolas widersprach der im Titel suggerierten zwangsläufigen Entwicklung „von Nietzsche zu Hitler“ - er wollte stattdessen Nietzsches „wahre Lehre“ nachzeichnen und sie angemaßten Auslegungen entgegenstellen. Dabei wandte er sich insbesondere gegen Julien Benda und dessen Einordnung Nietzsches unter die „Verräter“ in La Trahison des clercs $^{24}$. Ebenso wenig wie Nietzsche dafür verantwortlich gemacht werden dürfe, dass seine Philosophie missbraucht würde, dürften Intellektuelle die nationalsozialistischen Interpretationen Nietzsches unhinterfragt übernehmen. ${ }^{25}$ Jaspers seinerseits verzichtete - in seinem im Deutschen Reich verlegten Werk - auf eine explizit kritische Auseinandersetzung mit der nationalsozialistischen Vereinnahmung Nietzsches. Seine Grundsätze der Interpretation standen dieser jedoch diametral entgegen. So führe zu einem Verständnis Nietzsches „nicht die Hinnahme entschiedener Behauptungen als letzter Wahrheit [...], sondern der lange Atem, in dem man weiter fragt, Anderes und Entgegengesetztes hört, die Spannung der Möglichkeiten aufrechterhält" ${ }^{\text {26 }}$.

Den Paradoxien der Zeitläufte ist es zuzuschreiben, dass Heinrich Mann bei seiner Arbeit am Essay auch auf Werkausgaben Nietzsches zurückgriff, die Alfred Baeumler herausgegeben hatte. Bei seiner Flucht 1933 hatte er seine Bibliothek zurücklassen müssen und nun sandte ihm Golo Mann, der für den Band die Auszüge aus Nietzsches

21. Vgl. Steven E. Aschheim, Nietzsche und die Deutschen. Karriere eines Kults, übers. von Klaus Laermann, Stuttgart/ Weimar, Metzler, 1996, S. 292-315; Gerlach, „Politik (Faschismus, Nationalsozialismus, Sozialdemokratie, Marxismus)“, S. 504-509; Wolfgang Klein, „Nachwort“, in Nietzsches unsterbliche Gedanken, S. 173-188; Bernhard H. F. Tureck, Nietzsche und der Faschismus. Ein Politikum, Leipzig, Reclam, 2000, S. 116-121; Renate Werner, ,Nietzsche revisited. Zu Heinrich Manns Nietzsche-Essay von 1939“, Heinrich Mann-Jahrbuch 20, 2001, S. 141-158.

22. Vgl. Marius-Paulin Nicolas, De Nietzsche à Hitler, Paris, Fasquelle, 1936 (Akademie der Künste [AdK], Heinrich-Mann-Archiv [HMA], NB hm G 321). Das Buch wurde im Deutschen Reich sofort nach Erscheinen verboten.

23. Vgl. Karl Jaspers, Nietzsche. Einführung in das Verständnis seines Philosophierens, Berlin/ Leipzig, de Gruyter, 1936 (AdK, HMA, NB hm G 282).

24. Vgl. Julien Benda, La Trahison des clercs, Paris, Grasset, 1927 (AdK, HMA, NB hm B 1274).

25. Vgl. Nicolas, De Nietzsche à Hitler, S. (7)-21.

26. Jaspers, Nietzsche, S. 7. 
Werken zusammengestellt hatte, eben jene Ausgaben als Arbeitsgrundlage. ${ }^{27}$ In der mitgesandten Anthologie Nietzsches Philosophie in Selbstzeugnissen erklärte Baeumler: „Nicht die Vielfältigkeit der Nietzscheschen Begabung soll hier gezeigt werden, sondern die Einheit seines Denkens. [...] Wer diese Auswahl gelesen hat, wird nicht mehr bestreiten, daß Nietzsche ein philosophisches Weltbild von reiner Geschlossenheit hervorgebracht hat.“28 Golo Mann nahm hierauf Bezug, als er seinem Onkel über seine Zusammenstellung schrieb: „Leicht war es nicht, wenn man nämlich keinen halben, ungerechten, apodiktischen, undialektischen Nietzsche geben will, wie Bäumler [sic] es tut, und wie es allerdings sehr leicht ist." ${ }^{29}$ Für seine Auswahl hielt er fest: „Auf die Kritik der Kultur, Deutschlands, der Politik, lege ich das Schwergewicht; das hat heute die furchtbarste Aktualität." ${ }^{\text {"30 }}$ Diese bewusste Akzentuierung von Nietzsches Philosophie sollte die Differenzen zur nationalsozialistischen Politik offenlegen. Einen solchen kontrastiven Ansatz verfolgte auch Heinrich Mann in seinem einleitenden Essay.

\section{Heinrich Manns Nietzsche-Essay}

Ausgehend von seiner eigenen frühen Nietzsche-Faszination ${ }^{31}$ und der hier skizzierten Rezeptionsgeschichte stellt Heinrich Mann gleich zu Beginn seines Essays fest: „Sein Werk ist furchtbar, es ist bedrohlich geworden, anstatt dass es uns hinrisse wie vor Zeiten.“ (9) ${ }^{32}$ Als mögliche Verfahrensweise erwägt er daraufhin: „Nietzsche aufspüren, ihn wieder entdecken, hiesse wahrhaftig, ihn nochmals mit den Augen des Zwanzigjährigen lesen, neu sein Gedanke, unbefleckt sein Ruhm.“ (10) Eine solche Trennung von Werk und Wirkung könnte die Nietzsche-Mythen unterlaufen und widerlegen. Der Konjunktiv zeigt jedoch bereits an, dass der Konflikt nicht werkimmanent zu befrieden ist. Auch die Wirkung muss bei der erneuten Lektüre berücksichtigt werden. Gegen die Realitäten an einem ursprünglichen Nietzsche festzuhalten, ist keine Option. So bezieht Heinrich Mann seine Position

27. Hierzu gehörten: Friedrich Nietzsche, Morgenröte. Gedanken über die moralischen Vorurteile; ders., Die fröhliche Wissenschaft. («La gaya scienza»); ders., Götzendämmerung. Der Antichrist. Gedichte; ders., Der Wille zur Macht. Versuch einer Umwertung aller Werte; alle: Leipzig, Kröner, 1930. Diese Bände von Kröners Taschenausgabe enthielten jeweils ein Nachwort sowie eine sechsseitige Kurzbiographie „Das Leben Friedrich Nietzsches“ von Alfred Baeumler. Zudem sandte Golo Mann: Nietzsches Philosophie in Selbstzeugnissen, Band I: Das System, hrsg. und mit einem Nachwort versehen von Alfred Baeumler, Leipzig, Reclam, [1931] sowie Nietzsche in seinen Briefen und Berichten der Zeitgenossen. Die Lebensgeschichte in Dokumenten, hrsg. von Alfred Baeumler, Leipzig, Kröner, [1932] (AdK, HMA, NB hm G 225).

28. Alfred Baeumler, „Nachwort“, in Nietzsches Philosophie in Selbstzeugnissen, S. 243.

29. Golo Mann an Heinrich Mann, 3. Mai 1938 (AdK, HMA 1753); Kursivierungen im Original unterstrichen von Heinrich Mann. Zusätzlich markierte Heinrich Mann die Stelle mit einem Kreuz am Rand.

30. Ibid. Kursivierungen im Original unterstrichen von Heinrich Mann.

31. Für einen Überblick vgl. Volker Riedel, „Zur Nietzsche-Rezeption Heinrich Manns“, in Steffen Dietzsch, Claudia Terne (Hrsg.), Nietzsches Perspektiven, Berlin/ Boston, de Gruyter, 2014, S. 305331.

32. Alle Seitenangaben beziehen sich auf die Ausgabe Nietzsches unsterbliche Gedanken. 
als Leser und die Verschiebung seiner eigenen Rezeption explizit mit ein, wenn er konstatiert, „dass unsere Einsichten zunehmen konnten in dem Maasse, wie sein Werk und Ruhm nachwuchsen über sein Grab hinaus" (11). Der spezifische Kontext von Nationalsozialismus und Exil ist hiermit abgesteckt.

Grundlegend bleibt Nietzsche für Heinrich Mann ein ,grosser Herr des Geistes“ (27), der in erster Linie ein ambivalenter Denker war: „Ohne den Zweifel wäre niemand der unerbittlich Wahrhafte, als den er sich begriff. Auch ohne die Widersprüche nicht. Er hielt mit Recht sehr viel auf seine Widersprüche." (12) In seinem Essay verfährt Heinrich Mann nach demselben Prinzip, wenn er gleich zu Beginn schreibt: „Mit Recht oder Unrecht hielt er sich für einzig.“ (11) In den folgenden Abschnitten wird zu sehen sein, wie Heinrich Mann diese Ambivalenz im Einzelnen für seine „publizistische Öffentlichkeitsstrategie“ nutzbar machte, in der „alle Argumentation dem Ziel untergeordnet war, Hitler zu schlagen“33.

\section{Nietzsche und das „Reich“}

Die von Baeumler und anderen betriebene Indienstnahme Nietzsches für die nationalsozialistische Staatslehre und den Mythos des „Reiches“ wehrt Heinrich Mann rundheraus ab. Ausgehend von Nietzsches Positionierung als radikaler Individualist und als „Gegner des Staates“ schlussfolgert Heinrich Mann: „,noch eher wäre er ein Anarchist, als ein ergebener Bürger des ,Reiches““ (9) - also eher ein Professor Unrat als ein Untertan. ${ }^{34}$ Beide Typen erwähnt Heinrich Mann hier nicht explizit, für die im letztgenannten Roman gestalteten Auswüchse des Nationalismus im Deutschen Kaiserreich nimmt er Nietzsche jedoch als geistesverwandten Kronzeugen in Anspruch:

Das „Reich“ rüstete; es verwandelte eine Nation, die Denker gehabt hatte, in einen „heroisch gestimmten Igel“. Es beförderte den Nationalismus: hier tritt bei Nietzsche der Nationalismus auf, wohl gemerkt als eine Krankheit. „Die krankhafte Entfremdung, welche der Nationalitätswahnsinn zwischen die Völker Europas gelegt hat und noch legt, “ - er nennt das eine „Zwischenakts-Politik“. Nationalismus und „Reich“ - man hört heraus, was er ihnen im Grunde vorzuwerfen hat: sie machen die Köpfe dumpf, sie machen sie in wütender Weise schwelgerisch. (35)

Auf ein konkretes Beispiel, das eindeutige Parallelen zur politischen Situation der späten 1930er Jahre aufweist, folgt hier eine fundamentale Kritik des Nationalismus und seiner Irrationalitäten. Belege dafür, dass Nietzsche auch gegen demokratisch verfasste Gesellschaften polemisierte, ließ Heinrich Mann hier außen vor. Sein Fokus lag darauf, mit Nietzsche eine Mythologisierung des nationalsozialistischen „Reichs“ zu unterminieren. Dies ist nicht zuletzt im Hinblick auf die angedachte

33. Stein, Heinrich Mann, S. 119f.

34. Vgl. Heinrich Mann, Professor Unrat oder Das Ende eines Tyrannen (1905), mit einem Nachwort von Rudolf Wolff und einem Materialienanhang, zusammengestellt von Peter-Paul Schneider, Frankfurt a. M., Fischer, 1989 („Aus dem Tyrannen war endgültig der Anarchist herausgebrochen.“, S. 211) und ders., Der Untertan (1918) mit einem Nachwort und Materialienanhang von Peter-Paul Schneider, Frankfurt a. M., Fischer, 1991. 
US-amerikanische Leserschaft zu verstehen. Aus der Perspektive des Exils wies Heinrich Mann zudem auf einen nicht nur graduellen, sondern existenziellen Unterschied zwischen dem Bismarck'schen und dem Hitler'schen Reich hin:

[Nietzsche] ist weder verfolgt worden, noch war ihm verboten zu schreiben, wär' es sogar gegen das ,Reich“. Das hat sich seither ausgewachsen, wie man sieht und verspüren muss auf immer vielfachere Art, geistig, leiblich, in- und ausserhalb des „Reiches“, das bald kein Ausserhalb mehr zulässt. (36)

Die hier benannte akute Gefahr bestand unabhängig vom erfolgreich dekonstruierten Mythos fort. Noch im Jahr des Erscheinens von Heinrich Manns Essay begann das Deutsche Reich den Zweiten Weltkrieg, wurde die Prophezeiung Realität. Ein Jahr später war Frankreich besetzt und Heinrich Mann floh über Spanien und Portugal in die USA, wo die „Living Thoughts“-Reihe konzipiert und verlegt worden war.

\section{Nietzsche und die Gewalt}

Für die ideologisch-politische Vorbereitung des Krieges war im Nationalsozialismus unter anderem auf einen mythologisierten Begriff von Nietzsches „Willen zur Macht“ zurückgegriffen worden. In Heinrich Manns Essay taucht er nur kurz auf. Die Erwähnung ist auf der Kontextebene dennoch aufschlussreich. Heinrich Mann bezeichnet die gleichnamige Aphorismensammlung als Nietzsches „Hauptwerk, sein[] letzte[s] Wort“" (36). Interessant ist hier weniger, dass die Forschung das Werk mittlerweile als willkürliche Kompilation von Nachlass-Fragmenten - einen Mythos per se - erkannt hat, sondern vielmehr, dass Heinrich Mann diese Einschätzung aus Baeumlers Text „Das Leben Friedrich Nietzsches“ aus der von Golo Mann gesandten Kröner-Taschenausgabe exzerpiert hatte. ${ }^{35}$ Während Heinrich Mann aus Baeumlers Nachworten nichts notierte, sie wahrscheinlich nicht einmal las, war er offenbar davon ausgegangen, dass eine Kurzbiographie weniger anfällig für Mythologisierungen sei.

Den im Nationalsozialismus mit diesem Theorem verbundenen Gewaltmythos thematisierte Heinrich Mann in einem anderen Abschnitt seines Essays unter der Überschrift „Das Jasagen“. Dabei stellte er darauf ab, dass Nietzsches „Bekenntniss zur Macht in all ihrem Stumpfsinn“ (27) in erster Linie aus dessen individueller Selbstüberwindung resultiere: „Sein Jasagen - er ist dazu gekommen auf der Flucht vor seinem Leiden, in die fremde Welt der Herren dieser Erde, und was für Herren.“(36) Zugleich lässt er diese These nicht als mildernden Umstand gelten, wenn er Nietzsche seine „historische Schuld“36 zuweist: „Und er hat beigetragen. Er hat, wenn es um die Entscheidung über seine ,Werte' gehen sollte, für den Krieg gestimmt, ausdrücklich für Kriege mit sehr vielen Opfern.“ (29)

35. Vgl. Alfred Baeumler, „Das Leben Friedrich Nietzsches“, in Nietzsche, Götzendämmerung, S. 611 und Heinrich Mann, [Notizen zum Nietzsche-Essay], 40 Blatt, 48 Seiten (FML, Heinrich Mann Collection, Box 12, File 1b, alle Rechte vorbehalten S. Fischer Verlag GmbH, Frankfurt a. M.).

36. Werner, „Nietzsche revisited“, S. 150. 
Heinrich Manns Verfahrensweise in diesem Zusammenhang stellt überwiegend darauf ab, Nietzsches Postulate mit deren „nazistische[r] Konkretisation“37, also mit den gewalttätigen Realitäten, zu konfrontieren. Teilweise geschieht dies sarkastisch. So kommentiert er Nietzsches Forderungen nach ärztlicher Kontrolle von Eheschließungen und nach Kastration ,alle[r], die den Wert des Lebens zu verdächtigen suchen“ mit einem Hinweis auf nationalsozialistische Praktiken: „,keine Sorge, sie beeilen sich“. Zu Nietzsches Gebot, man müsse „,von den Kriegen her lernen, den Tod in die Nähe der Interessen zu bringen, für die man kämpft"،, merkt er an: „Wahrscheinlich verdient Ehrfurcht der Waffen- und Todeshändler Zaharoff“" Derlei Äußerungen Nietzsches sind für Heinrich Mann nicht entschuldbar, denn „diese Spielart [...] hätte dem Philosophen ins Auge fallen müssen, sie war sichtbar genug schon zu seinen Zeiten“ (30f.).

Um einer umfassenden Indienstnahme von Nietzsches Äußerungen für nationalsozialistische Gewaltmythen entgegenzuwirken, gesteht Heinrich Mann dem Philosophen dann allerdings doch zu, die Konsequenzen seines Denkens nicht in Gänze vorhergesehen zu haben: „Bei ihm sind die Starken und Vornehmen nun einmal tapfer, wie wären sie schon für ihn die uns vertrauten, erpresserischen Feiglinge gewesen.“ Zugleich kritisiert er Nietzsche für das Außerachtlassen historischer und sozialer Bedingungen in seiner Philosophie: „Die Frage hat er nie gestellt, woher die Starken und Vornehmen seines Sinnes in aller Welt noch kommen könnten." (29f.)

In Bezug auf die von Nietzsche geforderte Wiederherstellung der „Rangordnung“, in der obenan der geistige Adel stehe, kommentiert Heinrich Mann: „aber gerade der, je echter sein ,Geblüt', gerät nach unten, wo die Gewalt herrscht und übergreift. Nach dem geschehenen Umsturz der Werte erscheint es einfach, das zu wissen.“(29) Der letzte Satz bietet wieder die Möglichkeit, Nietzsche zu entlasten. Gleichzeitig würde die von Mann im Exil mehrfach thematisierte Intellektuellenfeindlichkeit des Nationalsozialismus ${ }^{38}$ auch vor Nietzsche nicht Halt machen:

Er hätte festgestellt und erfahren, dass die Forderung „Erkenne oder geh zugrunde!“ niemals an ihn gerichtet worden wäre. Die wirkliche Forderung heisst: „Geh zugrunde mit allen anderen! Geh als Erster zugrunde, sobald wir Starken und Vornehmen deine Erkenntniss zu fürchten haben! Wir Starken und Vornehmen fürchten alles, aber am meisten die Erkenntniss.“ (30)

Der hier herausgearbeitete Unterschied zwischen geistiger und körperlicher Stärke als konstitutiv für die Unterscheidung zwischen Nietzsches Begriff und dem nationalsozialistisch okkupierten lässt Heinrich Mann zu dem Schluss kommen, Nietzsches „Hass und Abscheu gälten dem gesamten Wachstum und Vollzug seines ausgebrüteten Unheils, und jedem, der sich auf ihn beruft“". Dies gelte insbesondere für „,den Typ, der seither in den Besitz der Gewalt gebracht worden ist, auch von ihm, leider auch von ihm“. Heinrich Mann entscheidet an dieser Stelle: „Aus wär' es mit seiner Neugier auf die ,starre Disciplin', auf ,Gewalt und List'. Seine ,blonde

37. Ibid., S. 149.

38. Vgl. u. a. Heinrich Mann, „L'Intelligence humiliée | Die erniedrigte Intelligenz“ (1933), in ders., Essays und Publizistik, Bd. 6, S. 91-108. 
Bestie' bliebe ihm in der Kehle stecken [...]. Er würde von seinem ,Jasagen“ nicht vieles noch einmal sagen." (31)

Das von Heinrich Mann hier verwendete rhetorische Stilmittel einer anachronistischen Annahme im Konjunktiv - kombiniert mit der klaren Zuweisung von historischer philosophischer Verantwortung - hat die Funktion, den Bezug nationalsozialistischer Gewaltmythen auf Nietzsche als grundsätzlich illegitim zu markieren. Ein Hinweis auf seine eigene Inanspruchnahme der „blonden Bestie“ für die ,deutsche[] Rasse“ ${ }^{\text {“39 }}$ von 40 Jahren zuvor schien ihm an dieser Stelle - trotz der zu Beginn des Essays erwähnten eigenen Rezeptionsverschiebungen - nicht angebracht.

Für geboten hielt er es dann allerdings, diesem abzulehnenden Modus politischer Herrschaft einen positiven strategischen Mythos entgegenzusetzen. Ausgehend davon, dass Gleichnisse in diesem Bereich grundsätzlich „den höheren Schichten des Menschentumes entnommen“ sein sollten, sei der von Nietzsche ins Spiel gebrachte Cesare Borgia die falsche Wahl, habe er doch „,seinen Platz allenfalls in einer hinteren Reihe. Seine Gewissenlosigkeit gab ein vollendetes Beispiel, aber sie führte zu nichts. “Mit dieser Einschätzung revidiert Heinrich Mann nochmals eigene frühere Urteile ${ }^{40}$ und stellt als Paradox hin: „In diese Gestalt eines unglücklichen Abenteurers hat der Erdichter des Übermenschen sich vergafft.“(32)Als „Abenteurer“ bezeichnete Heinrich Mann regelmäßig auch die Nationalsozialisten. ${ }^{41}$

Als Antipoden zu diesem Typus präsentiert er den von ihm im Exil porträtierten französischen König Henri Quatre: ${ }^{42}$ „Der ist der Fürst der Renaissance, und ist es allein.“ Er habe „mit seinem Volk“ das derzeitige Frankreich geschaffen, „Demokrat, der er schon war, und neigte zum Sozialismus schon hin“ (34). Heinrich Mann ergänzt hier sein seit der Jahrhundertwende wesentlich jakobinisch bestimmtes Frankreichbild ${ }^{43}$ um zwei Figuren aus dem Zeitalter des Humanismus. ${ }^{44}$ Die Verbindung zu Nietzsche sah er dadurch gegeben, dass Henri Quatre „der unmittelbare Schüler und Freund desselben Montaigne [war], der aus weiter Entfernung auf den Philosophen der Macht noch eingewirkt hat" (33). Doch um die tatsächliche Rezeption des Skeptikers Montaigne durch Nietzsche ist es ihm nicht zu tun. Vielmehr greift Heinrich Mann auf sein aktivistisches Paradigma von

39. Ders., „Zum Verständnisse Nietzsches“ (1896), in ders., Essays und Publizistik, Bd. 1: Mai 1889 bis August 1904, hrsg. von Peter Stein unter Mitarbeit von Manfred Hahn und Anne Flierl, Bielefeld, Aisthesis, 2013, S. 338-344.

40. Vgl. Ibid.

41. Vgl. Mann, Essays und Publizistik, Bd. 6, S. 57, 90, 116, 165, 175, 185, 368.

42. Vgl. Heinrich Mann, Die Jugend des Königs Henri Quatre (1935) und Die Vollendung des Königs Henri Quatre (1938), mit einem Nachwort von Hans Mayer und einem Materialienanhang, zusammengestellt von Peter-Paul Schneider, Frankfurt a. M., Fischer, 1991.

43. Vgl. Michaela Enderle-Ristori, „Das andere Frankreich. Exilerfahrungen Heinrich Manns“, in Jahrbuch für Internationale Germanistik, Reihe A, Bd. 82: Akten des XI. Internationalen Germanistenkongresses Paris 2005, Bd. 6: Migrations-, Emigrations- und Remigrationskulturen, Bern, Lang, 2007, S. 61-66.

44. Zur Humanismus-Thematik im Exil vgl. Thomas Koebner, ,'Militanter Humanismus'. Ein Konzept des dritten Weges im Exil"“, in: ders., Unbehauste. Zur deutschen Literatur in der Weimarer Republik, im Exil und in der Nachkriegszeit, München, edition text + kritik, 1992, S. 237-260. 
„Geist und Tat" zurück, das er in seinem gleichnamigen Essay ${ }^{45}$ formuliert hatte. Modellhaft für Deutschland stellte er darin Frankreich als Idealtypus einer Nation auf, in der Intellektualität und Macht keine Antagonismen seien. Dies tut er jetzt erneut im Exil: Anders als Cesare Borgia habe Henri Quatre Montaignes Diktum befolgt, ein Fürst „leuchte von Menschlichkeit, Wahrheit, Treue, Mässigung und besonders Gerechtigkeit: seltene Merkmale, verkannt und verbannt. [...] Nichts ist volkstümlich wie Gutsein.“ (33)

In dieser Absage an gewalttätige Herrschaft fungiert der „gute“ König Henri Quatre als „leuchtendes“ Beispiel. Vergleichend ordnet Heinrich Mann dabei Nietzsches Affirmation der Gewalt ein: „Das Jasagen zum Leben kann auch diese Laute haben, und sie zeugen von einem Geist, der einig mit dem Herzen ist. Nietzsche vergewaltigte sein Herz, sein Ja klingt schrill.“ (33)

Ein solch zweideutiger Gewaltbejaher Nietzsche dürfte kaum als mythische Bezugsfigur der nationalsozialistischen Staatslehre in Frage kommen. Heinrich Mann untermauert seine Argumentation an anderer Stelle, ausgehend von einem Zitat: „,Die Kultur und der Staat sind Antagonisten. “ [...] Man höre diesem Nietzsche eindringlicher zu, als dem, der anders spricht. [...] Denn die sonst gerühmte Härte wird auf einmal zum Verwerflichsten, wollte eine ungeistige Macht sich anmassen, den Gedanken zu versklaven." (35) Dieses Primat des Geistes erlaubt es Heinrich Mann, Nietzsche letztlich zugute zu halten, dass dieser ,den Schritt von der geistigen zur physischen Gewalt nie tat und nie getan hätte“47.

\section{Nietzsches „Übermensch“}

In seinem Abschnitt zum „Jasagen“ thematisiert Heinrich Mann auch Nietzsches Theorem des „Übermenschen“. Der Philosoph sei ,in seinen harten Gedanken bis zur Vernichtung des Menschengeschlechtes [gegangen], damit ein ,Übermensch` es ablöste, was eine Metaphysik des Diesseits vorstellt und ihm, aber sonst keinem, wohl ansteht" (32). Erneut verfolgt Heinrich Mann hier die Strategie, Nietzsches Begriff vom nationalsozialistischen Mythos zu entkoppeln, wobei er darüber hinweggeht, dass dessen „Übermensch“ kein abgeschlossener Zustand, kein Typus ist, sondern ein stetiger Prozess.

Darauf, dass sich der im Nationalsozialismus biologistisch und rassistisch propagierte „Herrenmensch“ nirgends in Nietzsches Werk findet, weist Heinrich Mann ebenso wenig hin. Er verwendet den Begriff gar selbst: „Übrigens stellt sich die Frage, wer sein Herrenmensch der Zukunft wirklich wäre.“ (36) Seine Antwort auf die Frage ist in mehrfacher Hinsicht erstaunlich und kontrovers:

45. Vgl. Heinrich Mann, „Geist und Tat“ (1910/11), in ders., Essays und Publizistik, Bd. 2, S. 113-119.

46. Im Roman lautet der letzte Satz übersetzt: „Stärker ist die Güte.“ (Mann, Die Jugend des Königs Henri Quatre, S. 381). Auf Latein findet er sich zudem in Mann, Die Vollendung des Königs Henri Quatre, S. 268, 377.

47. Klein, „Nachwort“, S. 173. 
Er hat über den Arbeiter sich ausgesprochen - wenig sozialistisch, aber im Sinne der proletarischen Diktatur. „Aus der Zukunft des Arbeiters“, da wird nicht mehr gefragt oder gefackelt. „Arbeiter sollten wie Soldaten empfinden lernen. Ein Honorar, ein Gehalt, aber keine Bezahlung! Kein Verhältniss zwischen Abzahlung und Leistung! Sondern das Individuum, je nach seiner Art, so stellen, dass es das Höchste leisten kann, was in seinem Bereich liegt.“ / Weiter: „Die Arbeiter sollen einmal leben wie jetzt die Bürger; - aber über ihnen, sich durch Bedürfnisslosigkeit auszeichnend, die höhere Kaste: also ärmer und einfacher, doch im Besitz der Macht." $(36)^{48}$

Hier aktualisiert nun also Heinrich Mann - gegen die zeitgenössische Rezeption - ein Nietzsche-Theorem. Der zweite Teilsatz des ersten Satzes im Zitat lautete in der Handschrift ursprünglich: ,heut wär’ es reiner Bolschewismus“449. Ende der 1930er Jahre im französischen Exil verwendete Heinrich Mann diesen Begriff in erster Linie, um die Sowjetunion als - maßgeblich geistig bzw. „sittlich“ bestimmten - diametralen Gegensatz zum Nationalsozialismus zu kennzeichnen. Im Rahmen seiner publizistischen und politischen Bemühungen um die Schaffung einer Volksfront im Exil besetzte er dabei sowjetische Begriffe und Figuren positiv. ${ }^{50}$ Es könnte sich hier also um den Versuch handeln, eine strategische Verbindungslinie von Nietzsches „Übermensch“ zum kommunistischen Arbeitermythos herzustellen. Heinrich Mann änderte die Passage jedoch in die zitierte Fassung, nachdem Golo Mann ihm seine Einwände mitgeteilt hatte:

Das mit den Arbeitern ist, meiner bescheidenen Ansicht nach, nicht richtig. Hier ist Nietzsche wenigstens so doppeldeutig wie überall sonst [...]. Er hat über den Sozialismus furchtbare Dinge gesagt, nicht aperçu-mässig, sondern aus dem Centrum seines Denkens heraus. Wenn er sich dennoch mit Marx begegnet, so, glaube ich, nicht in der Arbeiterfrage [... ${ }^{51}$

Trotz eindeutiger Korrekturfahnen behielt der Druck in Mass und Wert die ursprüngliche „Bolschewismus“-Fassung bei. Heinrich Manns Differenzierung kam somit nicht zum Tragen. Und so klar wie in seinen Notizen wollte er im Essay offenbar nicht argumentieren: Nietzsches Aussage sei „,[n]ur anti-sozialistisch, insofern der Sozialismus den Arbeitern immer nur gepredigt hat, bereit zu sein, - für wann? Und für was? Das haben sie bis heute nicht." "52

Doch Golo Manns Einspruch bezog sich nicht nur auf philosophische und politische Unvereinbarkeiten, er wies seinen Onkel auch auf eine nicht unwesentliche Parallele hin: „die Arbeiter seien Herrenmenschen, sagt Dr. Ley von der ,Arbeitsfront “" ${ }^{\text {“53. }}$. Auch Baeumler hatte 1934 geäußert: „Das männliche Zeitalter, das Zeitalter der Arbeiter und Soldaten, das von Nietzsche vorausgesagt wurde, ist im Anbrechen." ${ }^{54}$ Diese nationalsozialistische Kombination des Herrenmenschen- mit einem anders besetzten Arbeitermythos ließ Heinrich Mann von seinem Gedanken allerdings nicht

48. Kursivierungen im Original unterstrichen von Heinrich Mann.

49. Heinrich Mann, „Nietzsche“; FML (Heinrich Mann Collection, Box 12, Nr. 57, 45 Blatt, 51 Seiten, alle Rechte vorbehalten S. Fischer Verlag GmbH, Frankfurt a. M.).

50. Zu der damit verbundenen Problematik und früheren Deutungen des „Bolschewismus“ bei Heinrich Mann vgl. Wolfgang Klein, ,Sowjetisches bei Heinrich Mann“, in Walter Delabar, Walter Fähnders (Hrsg.), Heinrich Mann (1871-1950), Berlin, Weidler, 2005, S. 311-346.

51. Golo Mann an Heinrich Mann, 9. November 1938; FML (Heinrich Mann Collection, Box 4, File 30).

52. Mann, [Notizen zum Nietzsche-Essay].

53. Golo Mann an Heinrich Mann, 9. November 1938.

54. Baeumler, „Nietzsche und der Nationalsozialismus“, S. 293. 
grundsätzlich Abstand nehmen. Eine Antwort an seinen Neffen ist nicht bekannt, aber seinem Bruder schrieb er, der Einwand sei

richtig, so weit der Sozialismus in Betracht kommt. Die Worte über die Zukunft der Arbeiter als Herrenklasse sind nicht sozialistisch. Ich mache mir auch nichts daraus, dass Ley und seine Spiessgesellen in betrügerischer Absicht etwas Ähnliches sagen. Aber ich sehe, dass hier die Stelle ist, die für ein Buch ergänzt werden müsste. Vorerst mag sie stehen bleiben. ${ }^{55}$

Die Stelle blieb nicht nur ohne Ergänzung stehen, sie fand ihren Weg - zusammen mit dem oben stehenden Zitat Nietzsches „Aus der Zukunft des Arbeiters“ - auch leicht abgewandelt - ,es ist vorweggenommener Bolschewismus“ - in einen Beitrag Heinrich Manns zu Lenins 15. Todestag im Januar 1939. Timofej Rokotov, Redakteur der Moskauer Monatsschrift Internacional'naja literatura, hatte ihn darum gebeten. Heinrich Mann kommentierte die Nietzsche-Zeilen: „Lenin würde diesen Sätzen gewiss zugestimmt haben. Er würde anerkennen, dass er selbst angefangen hatte sie in die Tat umzusetzen, und dass ihre Verwirklichung weitergeht." ${ }^{6}$ "Während Baeumler Nietzsche zu einem Vorläufer Hitlers umgedeutet hatte, setzt Heinrich Mann hier Lenin als Erben ein. Ausschlaggebend für diesen Konnex dürfte neben der strategischen Vorgehensweise Heinrich Manns auch sein von Antagonismen geprägtes Denken sein, auf dessen Grundlage er beide Figuren in die „Traditionslinie der progressiven Kräfte" ${ }^{\text {“57 }}$ einordnete. Wie Nietzsche hatte er Lenin schon zuvor zu jenen Geistesgrößen gezählt, deren Werk weiterlebe. ${ }^{58}$

Die oben skizzierte kommunistische Kritik an Nietzsche als philosophischem Wegbereiter des Faschismus führte auf sowjetischer Seite vorhersehbar dazu, dass Heinrich Manns Zeilen über Lenin dort nicht erschienen. Der Antwortbrief Rokotovs an Heinrich Mann wurde allerdings mehrmals inhaltlich, strukturell und stilistisch umgearbeitet. ${ }^{59}$ In den umfangreichen Entwürfen wird abgewogen zwischen der Rücksichtnahme auf einen „linksbürgerlichen“, also nichtkommunistischen antifaschistischen Intellektuellen als Bündnispartner und einer grundlegenden Kritik an dessen Position. Angesichts des unmittelbar lebensbedrohlichen Ausmaßes staatlicher Repressionen in der Sowjetunion zur damaligen Zeit war allen Beteiligten - neben der Redaktion der Internacional'naja literatura auch die Abteilung für Agitation und Propaganda beim ZK der KPdSU - erkennbar daran gelegen, eine falsche Wortwahl unter allen Umständen zu vermeiden. Die letztlich gefundenen Formulierungen gehen von einem „Mißverständnis“ aus und präzisieren dann:

55. Thomas Mann, Heinrich Mann, Briefwechsel 1900-1949, hrsg. von Hans Wysling, 3., erw. Ausg., 2. Aufl., Frankfurt a. M., Fischer, 2005, S. 279.

56. Heinrich Mann, „Lenin, fünfzehn Jahre nach seinem Tode“, in ders., Mut, Paris, Editions du 10 Mai, 1939, S. 145-146, hier: 146.

57. Werner, „Nietzsche revisited“, S. 154.

58. Vgl. Heinrich Mann, „Beitrag zu: Политики и писатели Запада и Востока о В. И. Ленине“, in ders., Essays und Publizistik, Bd. 3: November 1918 bis 1925, hrsg. von Bernhard Veitenheimer mit Vorarbeiten von Barbara Voigt, Bielefeld, Aisthesis, 2015, S. 231 und „Fünf Jahre nach dem Tode Lenins“, in ders., Essays und Publizistik, Bd. 4: 1926 bis 1929, hrsg. von Ariane Martin, Bielefeld, Aisthesis, 2018, S. 350.

59. Vgl. Rossijskij Gosudarstvennyj Arkhiv Literatury i Iskusstva (RGALI), Fond 1397, Opis 1, Delo 662, Blatt 54-64, 67-77; Fond 1397, Opis 5, Delo 21, Blatt 2-5 (dieses Konvolut der Abteilung für Agitation und Propaganda beim ZK der KPdSU wurde als ,geheim“ eingestuft). 
„Arbeiter sollen wie Soldaten empfinden lernen“, sagt Nietzsche. Im Sowjetlande, das den von Lenin gewiesenen Weg eingeschlagen hat, hat sich indessen das gerade Gegenteil ereignet: Unser „Soldat" - der Rotarmist - hat gelernt, wie ein revolutionärer Arbeiter zu denken, der auf einem besonders verantwortlichen und ehrenvollen Posten der allgemeinen Front steht. [...] „Kein Verhältnis zwischen Abzahlung und Leistung. “Im Lande der Sowjets gilt aber das Grundgesetz des Sozialismus: ,jeder nach seinen Fähigkeiten, jedem nach seiner Leistung“. [...] Ein Mensch, der alle seine Fähigkeiten und all seine Kraft in den Dienst der sozialistischen Gesellschaft stellt, erhält nicht nur ein materielles Aequivalent für seine Arbeit, sondern auch Ehre und Ruhm, wird zum Volkshelden. Ist dem Arbeiter der Sowjetunion etwa Bedürfnislosigkeit eigen? [...] Nein, die Menschen der Sowjetunion sind durchaus keine düsteren Stoiker, die der Zukunft wie einem Moloch dienen, dem alle Lebensfreude und alle Lebensbedürfnisse zu opfern sind. ${ }^{60}$

Heinrich Manns Versuch, Nietzsches widersprüchliche Aussagen über die Arbeiter mit der sowjetischen Position zu verknüpfen, traf hier nicht nur auf eine grundsätzliche Ablehnung von dessen Philosophie, sondern auch auf einen Willen zum eigenen Mythos. Aus Heinrich Manns Notizen zum Essay geht hervor, dass er diesbezüglich andere Vorstellungen hatte: „Der sozialistische Volksstaat wird nicht besonders ,weich', aber er wird ohne Bosheit sein. "61 Diese Differenzierung verknüpft die von Nietzsche postulierte „Härte“ mit dem von Montaigne postulierten „Gutsein“ - in diesem Sinne „neigte“ auch Henri Quatre, wie oben zitiert, „,zum Sozialismus schon hin“"(34).

In seinem Antwortschreiben an Rokotov führt Heinrich Mann dies nicht an, sondern betont statt dessen, Nietzsches Worte seien „,nur biographisch zu verstehen“ - als Resultat von dessen Selbstüberwindung. Dann legt er seine Motivation für die Aktualisierung des Mythos vom „Übermenschen“ offen: Er habe in seinem Essay „grosses Gewicht darauf gelegt, dass er [Nietzsche] den Arbeitern, die auf seine Zeit folgen, die Stellung der höheren Kaste zuteilt. Damit entzieht er sich ein für alle Mal dem Missbrauch, den die Nationalsozialisten mit ihm begehen möchten." Letztlich erkennt er jedoch Rokotovs „Einwand als berechtigt an. Mein Nietzsche-Citat ist nicht geeignet zur Veröffentlichung in der Sowjet-Union; Sie konnten das früher erkennen als ich." ${ }^{62}$

Auch in seinem Antwortschreiben geht Heinrich Mann nicht auf den Unterschied zwischen Nietzsches Begriff der „Kaste“ und dem marxistischen der „Klasse“ ein. Ob er diesen „mit Fleiß übersehen“ hat oder ob dies seinem „Konzept einer Wertelite" ${ }^{63}$ geschuldet ist, bleibt schwierig zu beantworten. Erkennbar ist Heinrich Manns Ansatz, den nationalsozialistisch usurpierten Mythos des „Übermenschen“ zu unterminieren, indem er, konträr zur damaligen Rezeption, versucht, diesen unter Zuhilfenahme von Nietzsches Aussagen zur „Zukunft des Arbeiters“ zu aktualisieren. Angesichts des begrifflichen und konzeptionellen Dilemmas konnte diese Strategie jedoch nicht aufgehen. So musste sein „Versuch, sittlich wegweisend

60. Timofej Rokotov an Heinrich Mann, 5. Februar 1939; (FML Heinrich Mann Collection, Box 7, File 11, 3 Blatt, 3 Seiten).

61. Mann, [Notizen zum Nietzsche-Essay]. Zum Begriff des „Volksstaats“ bei Heinrich Mann vgl. ders., Essays und Publizistik, Bd. 3, S. 443.

62. Heinrich Mann an Timofej Rokotov, 13. Februar 1939 (RGALI, Fond 1397, Opis 1, Delo 662, Blatt 82), alle Rechte vorbehalten S. Fischer Verlag GmbH, Frankfurt a. M.; Kursivierungen im Original unterstrichen.

63. Werner, „Nietzsche revisited“, S. 154. 
in die zeitgenössischen Auseinandersetzungen um Nietzsche einzugreifen" ${ }^{64}$ in diesem Fall misslingen.

\section{Fazit}

In seiner Auseinandersetzung mit nationalsozialistisch besetzten Nietzsche-Mythen entwickelt Heinrich Mann verschiedene Argumentationsstrategien: Wo möglich, wendet er Nietzsches Denken direkt gegen einen Mythos, um diesen zu widerlegen. Alternativ arbeitet er die Ambivalenz von Nietzsches Philosophie heraus, um deren Vereinnahmung zur Legitimierung politischer Gewaltherrschaft zu unterminieren. Strategisch setzt er darüber hinaus auf französische Gegenmythen und schlägt eine „bolschewistische“ Umdeutung des Mythos vom „Übermenschen“ vor.

Diese Vorgehensweise ist gleichzeitig mehr als eine strategische. Heinrich Mann bleibt bei seiner langjährigen Anerkennung Nietzsches als ,grand seigneur des Geistes“ (36) - und zwar nicht trotz, sondern wegen seiner Widersprüche. Er weist ihm zwar klar die Verantwortung für die Anschlussfähigkeit seiner gewaltbejahenden Aussagen an die nationalsozialistische Ideologie zu, hält aber gegen die nationalsozialistische Mythenbildung - fest: „Gleichwohl hat Nietzsche für möglich gehalten, dass über diese Welt dereinst die Erkenntniss herrscht. Seine Auszeichnung und sein Vorrang ist dies - nur dies." Zur Entmythologisierung stellt Heinrich Mann hier allerdings nicht auf einen gänzlich rationalen Begriff der Erkenntnis ab, sondern möchte sie - mit Nietzsche - als einen leidenschaftlichen Zustand" (14) verstanden wissen.

Als eine gelungene Kombination von Leidenschaft und Vernunft führt Heinrich Mann den ,guten“ König Henri Quatre ein - als strategischen Gegenmythos zur nationalsozialistischen Gewaltherrschaft. In der Figur von Montaigne als dessen Ratgeber greift er zudem auf sein seit der Jahrhundertwende entwickeltes Bild von französischen Denkern als „leuchtenden“ Beispielen intellektuellen Engagements zurück. Nicht zuletzt präsentiert er Nietzsche (insbesondere in Passagen, deren Fokus nicht auf Mythischem liegt und die daher hier nicht behandelt wurden) als französisch beeinflussten, übernationalen ${ }^{65}$ Denker, dessen autoritärer Gestus ,durch die historisch bedingte Position des Alleinstehens gegen den Ungeist" 66 - eine für Heinrich Mann typisch deutsche Konstellation - zu erklären sei.

Vor diesem Hintergrund lässt Heinrich Mann Nietzsche, den die nationalsozialistische Mythologie als einheitlichen Philosoph und Politiker ${ }^{67}$ konstruiert hatte, in seinem Essay nicht als politische Größe gelten, sondern nur als ambivalente geistige. Seine Anfechtung der nationalsozialistischen Umdeutung Nietzsches formuliert er in seinen Notizen noch klarer als im ausgearbeiteten Essay, ausgehend von einem Nietzsche-Zitat: ,,Die Deutschen selber haben keine Zukunft.

64. Klein, „Nachwort“, S. 174.

65. Vgl. Heinrich Mann, „Das Bekenntnis zum Übernationalen“, in ders., Essays und Publizistik, Bd. 5: 1930 bis Februar 1933, hrsg. von Volker Riedel, Bielefeld, Aisthesis, 2009, S. 359-384.

66. Stein, Heinrich Mann, S. 140.

67. Vgl. Baeumler, Nietzsche der Philosoph und Politiker. 
[...] Sie haben keine, soweit sie N. ins Politische übersetzen wollen." ${ }^{68}$ Die Stelle, an der Heinrich Mann selbst Nietzsches Gedanken ins Politische übersetzt, um den Nationalsozialisten einen Mythos zu entziehen, scheitert konsequenterweise. Seine Argumentation führt dort am weitesten, wo sie Nietzsches Ambivalenzen ernst nimmt. ${ }^{69}$

Den Gedanken der „ewige[n] Wiederkunft“ - und damit den einer mythischüberhistorischen Gültigkeit von Nietzsche und seiner Philosophie - verweist Heinrich Mann am Ende seines Essays spöttisch ins Reich der Mythen: „Der Satz des Essays von der Erhaltung der Energie verspricht den Toten allenfalls, dass aus ihrem Grabe ein Obstbaum wächst.“ In diesem Sinne ist der letzte Satz - „Er ruhe in Frieden." (43) - nicht als Verabschiedung von Nietzsches Denken als unfruchtbar zu verstehen, wohl aber als Historisierung Nietzsches als Philosoph des 19. Jahrhunderts, dessen Denken sich nicht als ideologisch-politische Grundlage für das 20. Jahrhundert eignet.

68. Mann, [Notizen zum Nietzsche-Essay].

69. Bei der Lektüre von Jaspers notierte er: „N. ist immer fragwürdig und erinnert uns selbst an unsere Fragwürdigkeit, unser fliessendes Dasein, unsere Unbefestigtheit, Ungesichertheit. Er ist der Denker, den man nie besitzt, und der uns am Dringlichsten auffordert, zu denken, d. h. zu fragen." (Mann, [Notizen zum Nietzsche-Essay]). Im Essay findet sich die Passage nicht. 